\title{
Front Matter: Volume 8862
}

, "Front Matter: Volume 8862," Proc. SPIE 8862, Solar Physics and Space Weather Instrumentation V, 886201 (11 October 2013); doi:

10.1117/12.2046511

SPIE Event: SPIE Optical Engineering + Applications, 2013, San Diego, California, SPIE. United States 


\title{
PROCEEDINGS OF SPIE
}

\section{Solar Physics and Space \\ Weather Instrumentation $V$}

\author{
Silvano Fineschi \\ Judy Fennelly \\ Editors
}

25-26 August 2013

San Diego, California, United States

Sponsored and Published by

SPIE 
The papers included in this volume were part of the technical conference cited on the cover and title page. Papers were selected and subject to review by the editors and conference program committee. Some conference presentations may not be available for publication. The papers published in these proceedings reflect the work and thoughts of the authors and are published herein as submitted. The publisher is not responsible for the validity of the information or for any outcomes resulting from reliance thereon.

Please use the following format to cite material from this book:

Author(s), "Title of Paper," in Solar Physics and Space Weather Instrumentation $V$, edited by Silvano Fineschi, Judy Fennelly, Proceedings of SPIE Vol. 8862 (SPIE, Bellingham, WA, 2013) Article CID Number.

ISSN: 0277-786X

ISBN: 9780819497123

Published by

SPIE

P.O. Box 10, Bellingham, Washington 98227-0010 USA

Telephone +1 3606763290 (Pacific Time) · Fax +1 3606471445

SPIE.org

Copyright @ 2013, Society of Photo-Optical Instrumentation Engineers.

Copying of material in this book for internal or personal use, or for the internal or personal use of specific clients, beyond the fair use provisions granted by the U.S. Copyright Law is authorized by SPIE subject to payment of copying fees. The Transactional Reporting Service base fee for this volume is $\$ 18.00$ per article (or portion thereof), which should be paid directly to the Copyright Clearance Center (CCC), 222 Rosewood Drive, Danvers, MA 01923. Payment may also be made electronically through CCC Online at copyright.com. Other copying for republication, resale, advertising or promotion, or any form of systematic or multiple reproduction of any material in this book is prohibited except with permission in writing from the publisher. The CCC fee code is 0277-786X/13/\$18.00.

Printed in the United States of America.

Publication of record for individual papers is online in the SPIE Digital Library.

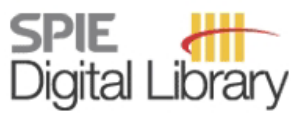

SPIEDigitalLibrary.org

Paper Numbering: Proceedings of SPIE follow an e-First publication model, with papers published first online and then in print and on CD-ROM. Papers are published as they are submitted and meet publication criteria. A unique, consistent, permanent citation identifier (CID) number is assigned to each article at the time of the first publication. Utilization of CIDs allows articles to be fully citable as soon as they are published online, and connects the same identifier to all online, print, and electronic versions of the publication. SPIE uses a six-digit CID article numbering system in which:

- The first four digits correspond to the SPIE volume number.

- The last two digits indicate publication order within the volume using a Base 36 numbering

system employing both numerals and letters. These two-number sets start with 00, 01, 02, 03, 04, $05,06,07,08,09,0 A, 0 B \ldots 0 Z$, followed by 10-1Z, 20-2Z, etc.

The CID Number appears on each page of the manuscript. The complete citation is used on the first page, and an abbreviated version on subsequent pages. Numbers in the index correspond to the last two digits of the six-digit CID Number. 


\section{Contents}

ix Conference Committee

\section{SESSION 1 SOLAR CORONAGRAPHS DESIGN AND OPTIMIZATION}

886204 Improved stray light suppression performance for the solar orbiter/METIS inverted external occulter [8862-3]

F. Landini, INAF - Osservatorio Astrofisico di Arcetri (Italy); M. Romoli, Univ. degli Studi di Firenze (Italy); G. Capobianco, INAF - Osservatorio Astrofisico di Torino (Italy); S. Vives, Lab. d'Astrophysique de Marseille, CNRS, Aix Marseille Univ. (France); S. Fineschi, G. Massone, D. Loreggia, INAF - Osservatorio Astrofisico di Torino (Italy); E. Turchi, Lab. Meccanico Enzo Turchi, Univ. degli di Firene (Italy); C. Guillon, C. Escolle, Lab. d'Astrophysique de Marseille, CNRS, Aix Marseille Univ. (France); M. Pancrazzi, M. Focardi, INAF - Osservatorio Astrofisico di Arcetri (Italy)

886205 Optimization of baffle configuration for stray light reduction [8862-4]

M. Pancrazzi, Lab. d'Astrophysique de Marseille, CNRS, Aix Marseille Univ. (France) and Univ. degli Studi di Firenze (Italy); S. Vives, Lab. d'Astrophysique de Marseille, CNRS, Aix Marseille Univ. (France); F. Landini, Univ. degli Studi di Firenze (Italy); C. Guillon, C. Escolle, J. Garcia, Lab. d'Astrophysique de Marseille, CNRS, Aix Marseille Univ. (France)

\section{SESSION $2 \quad$ ADVANCED OPTICS FOR SOLAR TELESCOPES}

886206 The high energy replicated optics to explore the sun mission: a hard x-ray balloon-borne telescope [8862-5]

S. D. Christe, A. Shih, M. Rodriguez, A. Cramer, K. Gregory, M. Edgerton, NASA Goddard Space Flight Ctr. (United States); J. Gaskin, C. Wilson-Hodge, J. Apple, K. Stevenson Chavis, A. Jackson, L. Smith, K. Dietz, B. O'Connor, A. Sobey, H. Koehler, B. Ramsey, NASA Marshall Space Flight Ctr. (United States)

886207 Solar alpha particles damage effects on UV and EUV optical coatings [8862-6]

M. Nardello, V. Polito, Univ. degli Studi di Padova (Italy); P. Zuppella, Istituto di Fotonica e Nanotecnologie (Italy); A. J. Corso, Univ. degli Studi di Padova (Italy); S. Zuccon, Istituto di Fotonica e Nanotecnologie (Italy); M. G. Sertsu, P. Nicolosi, Univ. degli Studi di Padova (Italy); S. Fineschi, INAF - Osservatorio Astrofisico di Torino (Italy); G. Naletto, Univ. degli Studi di Padova (Italy); E. Antonucci, INAF - Osservatorio Astrofisico di Torino (Italy); M. G. Pelizzo, Istituto di Fotonica e Nanotecnologie, CNR (Italy) 
886208 Test of a multilayer-coated EUV grating for I-IV order spectroscopic measurements of the solar corona [8862-33]

L. Poletto, F. Frassetto, P. Miotti, Istituto di Fotonica e Nanotecnologie, CNR (Italy); A. Giglia, Istituto Officina dei Materiali, CNR (Italy); A. J. Corso, P. Zuppella, M.-G. Pelizzo, Istituto di Fotonica e Nanotecnologie, CNR (Italy); S. Fineschi, E. Antonucci, Osservatorio Astronomico di Torino, INAF (Italy); G. Naletto, P. Nicolosi, Univ. degli Studi di Padova (Italy); M. Romoli, Univ. degli Studi di Firenze (Italy)

886209 Recent developments of ASPIICS: a giant solar coronagraph for the ESA/PROBA-3 formation flying mission [8862-34]

S. Vives, Lab. d'Astrophysique de Marseille, CNRS, Univ. Aix Marseille (France); J.-Y. Plesseria, Univ. de Liège (Belgium); P. Levacher, Lab. d'Astrophysique de Marseille, CNRS, Univ. Aix Marseille (France); W. Curdt, Max-Planck-Institut für Sonnensystemforschung (Germany); C. Guillon, Lab. d'Astrophysique de Marseille, CNRS, Univ. Aix Marseille (France)

8862 OA Development of multilayer coatings for solar orbiter EUV imaging telescopes [8862-35] F. Delmotte, E. Meltchakov, S. de Rossi, F. Bridou, A. Jérome, F. Varnière, R. Mercier, Lab. Charles Fabry, Institut d'Optique, CNRS, Univ. Paris Sud (France); F. Auchère, X. Zhang, B. Borgo, C. Dumesnil, S. François, Institut d'Astrophysique Spatiale (France); M. Roulliay, Institut des Sciences Moléculaires d'Orsay (France); U. Strauch, Helmholtz-Zentrum DresdenRossendorf (Germany)

\section{SESSION 3 ATST AND GROUND-BASED OBSERVATORIES}

8862 OB Solar chromosphere flare spectrograph [8862-7]

D. P. Choudhary, San Fernando Observatory, California State Univ., Northridge (United States); V. Yurchyshyn, Big Bear Solar Observatory (United States); S. Gosain, National Solar Observatory (United States)

8862 OC An off-limb solar adaptive optics system: design and testing [8862-8] G. E. Taylor, New Mexico State Univ. (United States); T. R. Rimmele, J. Marino, National Solar Observatory (United States); R. T. J. McAteer, New Mexico State Univ. (United States)

8862 OD The Advanced Technology Solar Telescope (ATST) project: a construction update (Invited Paper) [8862-9]

M. Warner, J. McMullin, T. Rimmele, T. Berger, National Solar Observatory (United States)

\section{SESSION 4 SOLAR ORBITER MISSION}

8862 OE The Solar Orbiter mission (Invited Paper) [8862-10]

D. Müller, European Space Research and Technology Ctr. (Netherlands); O. C. St. Cyr, NASA Goddard Space Flight Ctr. (United States) 
8862 OF SPICE EUV spectrometer for the Solar Orbiter mission (Invited Paper) [8862-11]

A. Fludra, D. Griffin, M. Caldwell, P. Eccleston, J. Cornaby, D. Drummond, W. Grainger,

P. Greenway, T. Grundy, C. Howe, C. McQuirk, K. Middleton, O. Poyntz-Wright, A. Richards,

K. Rogers, C. Sawyer, B. Shaughnessy, S. Sidher, I. Tosh, S. Beardsley, G. Burton, A. Marshall,

N. Waltham, S. Woodward, STFC Rutherford Appleton Lab. (United Kingdom);

T. Appourchaux, A. Philippon, F. Auchere, E. Buchlin, A. Gabriel, J.-C. Vial, Institut

d'Astrophysique Spatiale (France); U. Schühle, W. Curdt, D. Innes, S. Meining, H. Peter,

S. Solanki, L. Teriaca, Max-Planck-Institut für Sonnensystemforschung (Germany); M. Gyo,

V. Büchel, M. Haberreiter, D. Pfiffner, W. Schmutz, Physikalisch-Meteorologisches

Observatorium Davos (Switzerland); M. Carlsson, S. V. Haugan, Univ. of Oslo (Norway);

J. Davila, P. Jordan, W. Thompson, NASA Goddard Space Flight Ctr. (United States);

D. Hassler, B. Walls, C. Deforest, J. Hanley, J. Johnson, P. Phelan, Southwest Research Institute (United States); L. Blecha, H. Cottard, G. Paciotti, Almatech (Switzerland); N. Autissier, Y. Allemand, K. Relecom, APCO Technologies (Switzerland); G. Munro, A. Butler, ESR Technology Ltd. (United Kingdom); R. Klein, A. Gottwald, PhysikalischTechnische Bundesanstalt (Germany)

$88620 \mathrm{G}$ Novel space coronagraphs: METIS, a flexible optical design for multi-wavelength imaging and spectroscopy [8862-12]

S. Fineschi, E. Antonucci, INAF - Osservatorio Astrofisico di Torino (Italy); M. Romoli, Univ. degli Studi di Firenze (Italy); A. Bemporad, G. Capobianco, G. Crescenzio, G. Nicolini, G. Massone, D. Telloni, INAF - Osservatorio Astrofisico di Torino (Italy); M. Focardi, F. Landini, M. Pancrazzi, INAF - Osservatorio Astrofisico di Arcetri (Italy); L. Poletto, M.-G. Pelizzo, V. Da Deppo, IFN - CNR (Italy); J. D. Moses, U.S. Naval Research Lab. (United States); V. Andretta, INAF - Osservatorio Astronomico di Capodimonte (Italy); G. Naletto, P. Nicolosi, Univ. degli Studi di Padova (Italy); D. Spadaro, INAF - Osservatorio Astrofisico di Catania (Italy); A. Berlicki, Astronomical Institute of the ASCR, v.v.i. (Czech Republic); M. Uslenghi, INAF - IASF Milano (Italy); M. Malvezzi, Univ. degli Studi di Pavia (Italy);

L. Teriaca, Max-Planck-Institut für Sonnensystemforschung (Germany); L. Abbo, INAF Osservatorio Astrofisico di Torino (Italy); E. Magli, Politecnico di Torino (Italy)

$8862 \mathrm{OH}$ The solar and heliospheric imager (SoloHI) instrument for the solar orbiter mission (Invited Paper) [8862-13]

R. A. Howard, A. Vourlidas, C. M. Korendyke, S. P. Plunkett, M. T. Carter, D. Wang, N. Rich, U.S. Naval Research Lab. (United States); D. R. McMullin, Space Systems Research Corp. (United States); S. Lynch, A. Thurn, U.S. Naval Research Lab. (United States); G. Clifford, Silver Engineering, Inc. (United States); D. G. Socker, U.S. Naval Research Lab. (United States); A. F. Thernisien, George Mason Univ. (United States); D. Chua, M. G. Linton, U.S. Naval Research Lab. (United States); D. Keller, J. R. Janesick, J. Tower, M. Grygon, SRI International (United States); R. Hagood, W. Bast, ATK Space Systems (United States); P. C. Liewer, E. M. De Jong, M. M. C. Velli, Jet Propulsion Lab. (United States); Z. Mikic, Predictive Sciences Inc. (United States); V. Bothmer, Georg-August-Univ. Göttingen (Germany); P. Rochus, J. P. Halain, Univ. de Liège (Belgium); P. L. Lamy, Lab. d'Astrophysique de Marseille (France) 
8862 ol Seeing the corona with the solar probe plus mission: the wide-field imager for solar probe+ (WISPR) [8862-14]

A. Vourlidas, R. A. Howard, S. P. Plunkett, C. M. Korendyke, M. T. Carter, U.S. Naval Research Lab. (United States); A. F. R. Thernisien, George Mason Univ. (United States); D. H. Chua, U.S. Naval Research Lab. (United States); P. Van Duyne, Space Systems Research Corp. (United States); D. G. Socker, M. G. Linton, U.S. Naval Research Lab. (United States); P. C. Liewer, J. R. Hall, Jet Propulsion Lab. (United States); J. S. Morrill, U.S. Naval Research Lab. (United States); E. M. De Jong, Jet Propulsion Lab. (United States); Z. Mikic, Predictive Sciences Inc. (United States); P. Rochus, Univ. de Liège (Belgium); V. Bothmer, J. Rodman, Georg-August-Univ. Göttingen (Germany); P. Lamy, Lab. d'Astrophysique de Marseille (France)

$88620 \mathrm{~J}$ Development and test of an active pixel sensor detector for heliospheric imager on solar orbiter and solar probe plus [8862-15]

C. M. Korendyke, A. Vourlidas, S. P. Plunkett, R. A. Howard, D. Wang, U.S. Naval Research Lab. (United States); C. J. Marshall, A. Waczynski, NASA Goddard Space Flight Ctr. (United States); J. J. Janesick, T. Elliott, SRI International (United States); S. Tun, U.S. Naval Research Lab. (United States) and National Research Council (United States); J. Tower, M. Grygon, D. Keller, SRI International (United States); G. E. Clifford, Silver Engineering, Inc. (United States)

8862 OK Technology development for the Solar Probe Plus Faraday Cup [8862-16] M. D. Freeman, J. Kasper, A. W. Case, P. Daigneau, T. Gauron, J. Bookbinder, Smithsonian Astrophysical Observatory (United States); É. Brodu, Smithsonian Astrophysical Observatory (United States) and Lab. Procédés, Matériaux et Energie Solaire, CNRS (France); M. Balat-Pichelin, Lab. Procédés, Matériaux et Energie Solaire, CNRS (France); K. Wright, CSPAR, The Univ. of Alabama in Huntsville (United States)

8862 OL Mechanical design of the Solar Probe Cup instrument on Solar Probe Plus [8862-17] H. Bergner, D. Caldwell, A. W. Case, P. Daigneau, M. Freeman, J. Kasper, Smithsonian Astrophysical Observatory (United States)

$88620 \mathrm{M}$ The design, development, and implementation of a solar environmental simulator (SES) for the SAO Faraday Cup on Solar Probe Plus [8862-18]

P. Cheimets, J. Bookbinder, M. Freeman, R. Gates, T. Gauron, G. Guth, J. Kasper,

K. McCracken, W. Podgorski, Smithsonian Astrophysical Observatory (United States)

88620010 years of degradation trends of the SORCE SIM instrument [8862-20]

S. Béland, J. Harder, T. Woods, Univ. of Colorado at Boulder (United States) 
8862 OP Sounding rocket instrument development at Univ. of Alabama in Huntsville/NASA MSFC (Invited Paper) [8862-21]

K. Kobayashi, Univ. of Alabama in Huntsville (United States); J. Cirtain, A. Winebarger, S. Savage, NASA Marshall Space Flight Ctr. (United States); L. Golub, K. Korreck, HarvardSmithsonian Ctr. for Astrophysics (United States); S. Kuzin, P.N. Lebedev Physical Institute (Russian Federation); R. Walsh, Univ. of Central Lancashire (United Kingdom); C. DeForest, Southwest Research Institute (United States); B. DePontieu, A. Title, Lockheed Martin Space Systems Co. (United States); W. Podgorski, Harvard-Smithsonian Ctr. for Astrophysics (United States); R. Kano, N. Narukage, National Astronomical Observatory of Japan (Japan); J. Trujillo-Bueno, Instituto de Astrofisica de Canarias (Spain)

$88620 Q$ Effect of surface scatter upon the MTF of the solar ultra violet imager (SUVI) telescope (Invited Paper) [8862-22]

J. E. Harvey, Photon Engineering, LLC (United States); N. Choi, CREOL, The College of Optics and Photonics, Univ. of Central Florida (United States) and Photonic Systems Integrated, LLC (United States)

8862 OR The focusing optics $\mathbf{x}$-ray solar imager (FOXSI): instrument and first flight [8862-23] S. Krucker, Space Sciences Lab., Univ. of California, Berkeley (United States) and Univ. of Applied Sciences Northwestern Switzerland (Switzerland); S. Christe, NASA Goddard Space Flight Ctr. (United States); L. Glesener, Space Sciences Lab., Univ. of California, Berkeley (United States); S. Ishikawa, National Astronomical Observatory of Japan (Japan); B. Ramsey, M. Gubarev, NASA Marshall Space Flight Ctr. (United States); S. Saito, T. Takahashi, S. Watanabe, Institute of Space and Astronautical Sciences (Japan) and Univ. of Tokyo (Japan); H. Tajima, Nagoya Univ. (Japan); T. Tanaka, Kyoto Univ. (Japan); P. Turin, D. Glaser, J. Fermin, R. P. Lin, Space Sciences Lab., Univ. of California, Berkeley (United States)

8862 OS Design progress of the solar UV-Vis-IR telescope (SUVIT) aboard SOLAR-C [8862-24] Y. Katsukawa, National Astronomical Observatory of Japan (Japan); K. Ichimoto, Kwasan and Hida Observatory (Japan); Y. Suematsu, H. Hara, R. Kano, National Astronomical Observatory of Japan (Japan); T. Shimizu, K. Matsuzaki, ISAS, Japan Aerospace Exploration Agency (Japan)

8862 OT The soft $x$-ray photon-counting spectroscopic imager for the sun [8862-25] T. Sakao, N. Narukage, ISAS, Japan Aerospace Exploration Agency (Japan); M. Shimojo, National Astronomical Observatory of Japan (Japan); K. Watanabe, ISAS, Japan Aerospace Exploration Agency (Japan); Y. Suematsu, National Astronomical Observatory of Japan (Japan); S. Imada, Nagoya Univ. (Japan); S. Ishikawa, National Astronomical Observatory of Japan (Japan) 
8862 OU Recent advances in electronics and software for the METIS coronagraph aboard solar orbiter [8862-26]

M. Focardi, M. Pancrazzi, INAF - Osservatorio Astrofisico di Arcetri (Italy); G. Nicolini, INAF Osservatorio Astronomico di Torino (Italy); E. Magli, M. Ricci, Politecnico di Torino (Italy); M. Uslenghi, INAF - IASF Milano (Italy); M. Romoli, Univ. degli Studi di Firenze (Italy); F. Landini, INAF - Osservatorio Astrofisico di Arcetri (Italy); E. Antonucci, S. Fineschi, INAF Osservatorio Astronomico di Torino (Italy); G. Naletto, P. Nicolosi, Univ. degli Studi di Padova (Italy) and Istituto di Fotonica e Nanotecnologie, CNR (Italy); D. Spadaro, INAF Osservatorio Astrofisico di Catania (Italy); V. Andretta, INAF - Osservatorio Astronomico di Capodimonte (Italy); W. Errico, F. Bigongiari, L. Fontani, M. Orlandi, A. Colonna, Sitael S.p.a. (Italy)

8862 OW Detector and imaging systems for the gamma-ray imager/polarimeter for solar flares (GRIPS) instrument [8862-28]

N. Duncan, Space Sciences Lab., Univ. of California, Berkeley (United States); A. Shih, NASA Goddard Space Flight Ctr. (United States); G. Hurford, P. Saint-Hilaire, A. Zoglaver, H. Bain, Space Sciences Lab., Univ. of California, Berkeley (United States); M. Amman, Lawrence Berkeley National Lab. (United States); S. Boggs, R. Lin, Space Sciences Lab., Univ. of California, Berkeley (United States)

$88620 X$ Preliminary tolerance analysis of the coronagraphic instrument METIS for the Solar Orbiter ESA mission [8862-29]

V. Da Deppo, Istituto di Fotonica e Nanotecnologie, CNR (Italy); G. Crescenzio, INAF Osservatorio Astrofisico di Torino (Italy); G. Naletto, Univ. degli Studi di Padova (Italy) and Istituto di Fotonica e Nanotecnologie, CNR (Italy); S. Fineschi, INAF - Osservatorio Astrofisico di Torino (Italy); M. Romoli, Univ. degli Studi di Firenze (Italy); E. Antonucci, INAF Osservatorio Astrofisico di Torino (Italy)

$88620 \mathrm{Y}$ Characterization of linear polarizers in the wavelength range 100-150 nm (VUV) for solar physics applications [8862-30]

G. Capobianco, INAF - Osservatorio Astrofisico di Torino (Italy); M. Malvezzi, Univ. degli Studi di Pavia (Italy); S. Fineschi, INAF - Osservatorio Astrofisico di Torino (Italy); J. I. Larruquert, Instituto de Optica, CSIC (Spain); A. Giglia, Istituto Officina dei Materiali, CNR (Italy); J. A. Aznarez, Instituto de Optica, CSIC (Spain); G. Massone, G. Crescenzio, INAF Osservatorio Astrofisico di Torino (Italy); S. Nannarone, Univ. di Modena e Reggio Emilia (Italy); F. Frassetto, Istituto di Fotonica e Nanotecnologie, CNR (Italy); J. A. Mendez, L. Rodríguez-de Marcos, Instituto de Optica, CSIC (Spain); P. Miotti, Istituto di Fotonica e Nanotecnologie, CNR (Italy)

886210 The visible light source for METIS stray-light tests: preliminary design [8862-32] M. Tordi, Space Technologies S.r.I. (Italy); S. Fineschi, G. Crescenzio, INAF - Osservatorio Astrofisico di Torino (Italy)

Author Index 


\title{
Conference Committee
}

\author{
Program Track Chair
}

Oswald H. Siegmund, University of California, Berkeley (United States)

\section{Conference Chairs}

Silvano Fineschi, INAF - Osservatorio Astronomico di Torino (Italy)

Judy Fennelly, Air Force Research Laboratory (United States)

\section{Conference Program Committee}

Frédéric Auchère, Institut d'Astrophysique Spatiale (France)

Thomas R. Caudill, Air Force Research Laboratory (United States)

loannis A. Daglis, National Observatory of Athens (Greece)

Dominic B. Doyle, European Space Research and Technology Center (Netherlands)

Federico Landini, INAF - Osservatorio Astrofisico di Arcetri (Italy)

Toshifumi Shimizu, Japan Aerospace Exploration Agency (Japan)

Sébastien Vivès, Observatoire Astronomique de Marseille-Provence

(France)

Session Chairs

1 Solar Coronagraphs Design and Optimization

Silvano Fineschi, INAF - Osservatorio Astronomico di Torino (Italy)

2 Advanced Optics for Solar Telescopes

Silvano Fineschi, INAF - Osservatorio Astronomico di Torino (Italy)

3 ATST and Ground-based Observatories

Judy Fennelly, Air Force Research Laboratory (United States)

4 Solar Orbiter Mission

Silvano Fineschi, INAF - Osservatorio Astronomico di Torino (Italy)

Angelos Vourlidas, U.S. Naval Research Laboratory (United States)

5 Solar Probe Plus Mission

Silvano Fineschi, INAF - Osservatorio Astronomico di Torino (Italy)

Daniel Mueller, European Space Agency (Netherlands) 
6 Space Weather and Sun Monitoring Judy Fennelly, Air Force Research Laboratory (United States)

7 Solar Space Instrumentation Judy Fennelly, Air Force Research Laboratory (United States) Federico Landini, INAF - Osservatorio Astrofisico di Arcetri (Italy) 